\title{
Desenvolvimento de Chrysoperla externa alimentada na fase larval com ovos de Bonagota cranaodes
}

\author{
Development of Chrysoperla externa fed in the larval stage with eggs of Bonagota cranaodes
}

\author{
Ana Lúcia de Paula Ribeiro ${ }^{\mathrm{I}}$ Alessandro Dal'Col Lúcio ${ }^{\mathrm{II}}$ Ervandil Corrêa CostaII \\ Andreza Ribeiro Bolzan ${ }^{\mathrm{IV}}$ Rosmari Jovanowichs ${ }^{\mathrm{V}}$ Cinei Terezinha Riffel $^{\mathrm{VI}}$
}

\section{RESUMO}

\begin{abstract}
Este trabalho teve por finalidade realizar estudos biológicos da espécie de crisopídeo de maior ocorrência nos agroecossistemas brasileiros, Chrysoperla externa, a fim de viabilizar a criação desta em condições de laboratório. $O$ trabalho foi desenvolvido no Laboratório de Entomologia da Universidade Federal de Santa Maria, onde foi estudada a biologia das fases imatura e adulta de C. externa, alimentando suas larvas com ovos de Bonagota cranaodes e os adultos com dieta artificial a base de lêvedo de cerveja e mel na proporção de 1:1. O período embrionário foi determinado utilizando cápsulas de gelatina e tubos de vidro de $2,5 \times 8,5 \mathrm{~cm}$ e os insetos adultos foram criados em gaiolas de tubo de PVC com 10,0 $\mathrm{cm}$ de diâmetro e $23,0 \mathrm{~cm}$ de comprimento em temperatura de $25 \pm 2{ }^{\circ} \mathrm{C}$ e $70 \pm 10 \%$ de umidade relativa e fotofase de 14 horas. De acordo com os resultados obtidos, o período embrionário foi de 4,0 dias, com viabilidade de 89,2\%. Para a fase larval, foi determinada uma duração de 13,1 dias, sendo a duração média para o 1으. 2o e 3o instares de 3,7; 4,9; e 4,5 dias, respectivamente. $O$ período médio de pré-pupa e pupa foi de 1,4 e 9,3 dias, respectivamente, perfazendo um ciclo de desenvolvimento médio de 27,8 dias. As medidas da largura da cápsula cefálica mostraram que a regra de Dyar aplica-se nesse caso. Larvas de Chrysoperla externa podem ser criadas em ovos de Bonagota cranaodes em condições de laboratório.
\end{abstract}

Palavras-chave: controle biológico, crisopídeos, lagartaenroladeira.

\section{ABSTRACT}

The paper aimed to conduct biological studies of the chrysopidae species most frequent in the Brazilian agroecosystems, Chrysoperla externa, in order to raise this species under laboratory conditions. The research was developed at the Entomology Laboratory of the Federal University of Santa Maria, where biological studies of immature and adult stages of $\boldsymbol{C}$. externa were performed, feeding its larva with eggs of Bonagota cranaotes and adults with an artificial diet based on beer yeast and honey, in the 1:1 proportion. The embryonic period was determined using gel capsules and $2.5 \times 8.5 \mathrm{~cm}$ glass tubes. The adult insects were raised in PVC tubes of $10.0 \mathrm{~cm}$ diameter and $23.0 \mathrm{~cm}$ length, at the temperature of $25^{\circ} \mathrm{C} \pm 2{ }^{\circ} \mathrm{C}$ and $70 \pm 10 \%$ relative humidity and 14 hour photophase. According to the results obtained, the embryonic period was of 4.0 days, with $89.2 \%$ of viability, the mean larval period for the $1^{\text {st }}, 2^{\text {nd }}$ and $3^{\text {rd }}$ instars was of 3.7, 4.9 and 4.5 days, respectively. The mean period for pre-pupa and pupa was of 1.4 and 9.3 days, respectively, amounting to a mean developing cycle of 27.8 days. The width measures of the cephalic capsule showed that the Dyar's rule can applied to this case. Larva of Chrysoperla externa may be raised in eggs of Bonagota cranaodes under laboratory conditions.

Key words: biological control, chysopidae, leaf-roller.

\section{INTRODUÇÃO}

Uma das técnicas do manejo integrado de pragas (MIP) consiste na preservação dos agentes de controle biológico. CANARD \& PRINCIPI (1984) e FREITAS \&ADAMS (1994) citam os crisopídeos como

\footnotetext{
IUniversidade de Cruz Alta (UNICRUZ), 98020-290, Cruz Alta, RS, Brasil. E-mail: aldpr2008@gmail.com. Autor para correspondência.

IIDepartamento de Fitotecnia, Centro de Ciências Rurais (CCR), Universidade Federal de Santa Maria (UFSM), Santa Maria, RS, Brasil.

"IDepartamento de Defesa Fitossanitária, CCR, UFSM, Santa Maria, RS, Brasil.

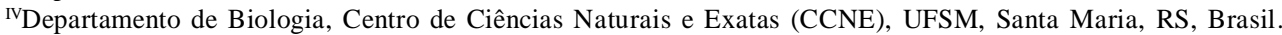

vCooperativa Agropecuária de Júlio de Castilhos (COTRIJUC), Júlio de Castilhos, RS, Brasil.

VISociedade Educacional Três de Maio (SETREM), Três de Maio, RS, Brasil.
} 
um dos mais eficientes predadores, cujas larvas alimentam-se vorazmente de pulgões, moscas brancas, ovos e pequenas lagartas de lepidópteros, de diversas cochonilhas e de ácaros.

A família Chrysopidae possui cerca de 1.200 espécies descritas, destacando-se como um dos grupos mais diversos. No Brasil, a fauna de crisopídeos conhecida totaliza aproximadamente 148 espécies, envolvendo representantes de todas as subfamílias e tribos de Chrysopidae, com exceção da tribo Ankylopterygini (ADAMS \& PENNY, 1987; PENNY, 1977; FREITAS \& PENNY, 2001; CARDOSO et al., 2003).

Muitas espécies de Chrysopidae são criadas em condições de laboratório, contornando alguns problemas como o canibalismo durante a fase de larva e produção de dietas inadequadas. Atualmente, o uso de ovos de Anagasta kuehniella (RIBEIRO, 1991), Sitotroga cerealella (ALBUQUERQUE et al., 1994; BIAGIONI \& FREITAS, 2001) Alabama argillacea (SILVA et al., 2002) e algumas espécies de pulgões (FONSECA et al., 2001; MAIA et al., 2000) permitem a criação deste predador.

Dados de literatura demonstram o potencial dos crisopídeos como agentes de controle biológico de pragas, em várias culturas de interesse econômico. NUÑEZ (1988) afirma que os crisopídeos são inimigos naturais promissores no MIP de culturas como milho, batata, algodão, citrus e maçã. FERREIRA et al. (2006) destaca a importância do uso dos crisopídeos no controle biológico da lagarta-enroladeira, Bonagota cranaodes, praga importante na cultura da macieira em pomares comerciais no sul do Brasil.

É possível a manipulação e o aumento de população de inimigos naturais já existentes na natureza via produção de agentes de controle biológico, por meio de criações em laboratório, seguido de liberações e colonizações no campo (FREITAS, 2002). Para isso, é necessário a produção em grande escala de insetos em laboratório, assim como o conhecimento sobre a bioecologia, hábitos alimentares e requisitos nutricionais dos inimigos naturais.

Dessa forma, o trabalho teve por objetivo estudar a biologia de Chrysoperla externa considerada a espécie de crisopídeo de maior ocorrência em cultivos de alface hidropônica, a fim de viabilizar a sua criação em condições de laboratório quando alimentadas com ovos de Bonagota cranaodes.

\section{MATERIAL E MÉTODOS}

Os ovos, as larvas, as pupas e os adultos de crisopídeos foram coletados na área experimental do Departamento de Fitotecnia da Universidade Federal de Santa Maria - UFSM, com coordenadas geográficas: $53^{\circ} 48^{\prime} 42^{\prime \prime}$ (latitude oeste) e $29^{\circ} 41^{\prime} 25^{\prime \prime}$ (latitude sul) e $95 \mathrm{~m}$ de altitude. A alface foi cultivada em sistema hidropônico utilizando a cultivar 'Vera' .

A criação de crisopídeos foi realizada no laboratório de Entomologia do Departamento de Defesa Fitossanitária da UFSM, com início no mesmo período do cultivo da alface. As larvas foram alimentadas em todas as etapas do estudo com ovos de Bonagota cranaodes, tendo como base a metodologia desenvolvida por PARRA et al. (1995), e enviadas para oLaboratório de Entomologia da Universidade Estadual Norte Fluminense em Campos Goytacazes, para identificação.

Para obtenção da geração $F_{1}$, adotou-se a técnica desenvolvida por FREITAS (2001) de criação dos adultos de crisopídeos em laboratório, onde os insetos adultos foram alimentados com lêvedo de cerveja e mel na proporção de 1:1. Após a eclosão, as larvas foram individualizadas em tubos de vidro de $2,5 \mathrm{~cm}$ de diâmetro por $8,5 \mathrm{~cm}$ de altura e tamponados com filme de PVC, mantidos em câmaras climatizadas tipo BOD com temperatura de $25 \pm 2^{\circ} \mathrm{C}$ e $70 \pm 10 \%$ de umidade relativa e fotofase de 14 horas.

Os adultos recém-emergidos foram separados, formando-se dez casais, sendo que cada casal foi colocado em gaiola individual de tubo de PVC com $10,0 \mathrm{~cm}$ de diâmetro e $23,0 \mathrm{~cm}$ de comprimento, acondicionado em câmara BOD com temperatura de $25 \pm 2^{\circ} \mathrm{Ce} 70 \pm 10 \%$ de umidade relativa e fotofase de 14 horas para os estudos biológicos e a manutenção da criação. Os tubos foram devidamente identificados por números, geração correspondente $\left(\mathrm{F}_{2}\right)$, data da montagem, dados referentes à fêmea e ao macho e a data do início de oviposição. Foram individualizados 900 ovos da geração $F_{2}$ os quais foram acondicionados em cápsulas de gelatina até o momento da eclosão, sendo as larvas transferidas para tubos de vidro e alimentadas com ovos de $\boldsymbol{B}$. cranaodes.

O estudo da biologia das fases imaturas foi conduzido com avaliações diárias desde a postura até a emergência dos adultos. As variáveis avaliadas foram a duração e a viabilidade das fases de desenvolvimento e do ciclo biológico (ovo-adulto). Para a estimativa da duração da fase de pré-pupa, foi considerado o intervalo entre o início da construção do casulo e a última ecdise larval. Por meio de uma ocular graduada, acoplada a um microscópio estereoscópico, mediu-se a largura da cápsula cefálica de 42 larvas, em cada um dos instares, adotando-se o método de distribuição de frequências, proposto por PARRA \& HADDAD (1989).

Para a fase adulta de Chrysoperla externa, os estudos foram realizados observando-se dez casais 
da geração $F_{3}$ provenientes das larvas utilizadas nos estudos da biologia das fases imaturas. Os casais foram formados com um intervalo máximo de $24 \mathrm{~h}$ de diferença entre a emergência de machos e fêmeas. As avaliações foram realizadas durante 50 dias (período máximo de sobrevivência dos adultos em laboratório), sendo avaliado o período de pré-oviposição, a fecundidade diária e total, o período de oviposição, o número mínimo e máximo de ovos por dia e a longevidade de fêmeas e machos. Para avaliar o consumo de C. externa em ovos de B. cranaodes, foram individualizadas 20 larvas de C. externa em tubos de vidro, disponibilizando ovos diariamente.

No estudo da tabela de vida de fertilidade de $\boldsymbol{C}$. externa, foram utilizados 20 casais colocados nos tubos de PVC, acondicionados em câmaras climatizadas nas mesmas condições da criação de manutenção. Foi avaliada a razão sexual ( $\mathrm{rs}=\mathrm{n}^{\mathrm{o}}$ fêmeas/ no machos $+n^{\circ}$ fêmeas), a mortalidade diária de machos e fêmeas, a duração dos períodos de pré-oviposição e oviposição, o número médio de ovos em cada data de oviposição e a viabilidade de ovo a adulto.

A partir dos dados de sobrevivência e oviposição de cada fêmea, foi elaborada a tabela de vida de fertilidade. Estimou-se o número médio de ovos por fêmea $(\mathrm{mx})$ em cada data de oviposição $(\mathrm{x})$, considerando o total de fêmeas, o índice de sobrevivência acumulado de fêmeas (lx) durante o período de oviposição e o número de descendentes que atingiram a idade $x$ na geração seguinte (lx.mx), seguindo a metodologia de SILVEIRA NETO et al. (1976). A partir destes resultados, definiu-se a tabela de vida e estimaram-se a duração média de gerações (T), a taxa líquida de reprodução (Ro), a taxa intrínseca de crescimento $\left(r_{m}\right)$ e a taxa finita de crescimento (l).

\section{RESULTADOS E DISCUSSÃO}

Foram identificadas duas espécies de crisopídeos. Uma apresentava abdome com pequenas cerdas e foi identificada como Chrysoperla externa, enquanto que a outra, com cerdas longas no abdome que permitem reter restos de exúvia, foi identificada como Ceraeochrysa sp. Como o maior número de indivíduos que compuseram a coleta de crisopídeos foi C. externa ( $85 \%$ do total), esta foi selecionada para o estudo da biologia a fim de viabilizar a sua criação em condições de laboratório, quando alimentada com $\boldsymbol{B}$. cranaodes.

O período embrionário de $\boldsymbol{C}$. externa, durante a geração $F_{2}$, foi de 4,0 dias e a viabilidade de $89 \%$ (Tabela 1). Este resultado corrobora os obtidos em trabalhos realizados no Brasil por MAIA et al. (2000) e AUAD et al. (2005) sobre a mesma espécie, evidenciando que $\boldsymbol{C}$. externa possui um comportamento homogêneo dentro de um ambiente controlado, apesar da procedência dos indivíduos coletados serem diferentes.

A duração média do $1^{\circ}$ - ínstar foi de 3,7 dias e a viabilidade de $96,7 \%$ (Tabela 1). Esta viabilidade ficou próxima ao valor de $97 \%$ obtido por CARVALHO et al. (1998) para larvas de C. externa alimentadas com ovos de Alabama argillacea, mantidas a $24^{\circ} \mathrm{C}$. De acordo com CANARD \& PRINCIPI(1984), a viabilidade,

Tabela 1 - Número de observações (n), limites mínimo (LMN) e máximo (LMX), duração média, em dias, desvio padrão (DP) e viabilidade (\%) das fases imaturas e adultas de Chrysoperla externa em ovos de Bonagota cranaodes com temperatura de $25 \pm 2{ }^{\circ} \mathrm{C}$ e $70 \pm 10 \%$ de umidade relativa e fotofase de 14 horas.

\begin{tabular}{|c|c|c|c|c|c|}
\hline Parâmetros Biológicos & $\mathrm{N}$ & LMN & LMX & Média \pm DP & Viabilidade $\%$ \\
\hline \multicolumn{6}{|l|}{ Fases imaturas } \\
\hline Período embrionário dias & 900 & 2,00 & 6,00 & $4,0 \pm 0,89$ & 89,2 \\
\hline $1^{\circ}$ ínstar larval & 803 & 2,00 & 6,00 & $3,7 \pm 0,79$ & 96,7 \\
\hline $2^{\circ}$ ínstar larval & 777 & 3,00 & 7,00 & $4,9 \pm 0,87$ & 100,0 \\
\hline $3^{\circ}$ ínstar larval & 777 & 3,00 & 6,00 & $4,5 \pm 0,97$ & 100,0 \\
\hline Pré-pupa (dias) & 674 & 1,00 & 2,00 & $1,4 \pm 0,50$ & 86,7 \\
\hline Pupa (dias) & 560 & 7,00 & 13,00 & $9,3 \pm 1,43$ & 83,1 \\
\hline \multicolumn{6}{|l|}{ Fase adulta } \\
\hline Pré-oviposição (dias) & --- & 1,0 & 3,0 & $2,1 \pm 1,06$ & --- \\
\hline $\mathrm{N}^{\mathrm{o}}$ de ovos total & --- & 31 & 444 & $163 \pm 153,48$ & --- \\
\hline Período de oviposição (dias) & --- & 16 & 49 & $33,6 \pm 11,67$ & --- \\
\hline $\mathrm{N}^{\mathrm{o}}$ de ovos dia ${ }^{-1}$ & --- & 0,0 & 23 & $11,3 \pm 2,69$ & --- \\
\hline № ovos fêmea ${ }^{-1}$ & --- & 0,8 & 11 & $6,0 \pm 4,54$ & --- \\
\hline Longevidade de fêmeas (dias) & --- & 17 & 54 & $38,4 \pm 11,53$ & --- \\
\hline Longevidade de machos (dias) & --- & 17 & 53 & $36,1 \pm 14,76$ & --- \\
\hline
\end{tabular}

Ciência Rural, v.41, n.9, set, 2011. 
nesse estádio, é normalmente alta se o inseto receber alimentação adequada. O 2o ínstar apresentou uma duração de 4,9 dias (Tabela 1). A viabilidade foi de $100 \%$, confirmando os resultados obtidos por CARVALHO et al. (1998) com C. externa mantida à temperatura de $24^{\circ} \mathrm{C}$ e alimentada com ovos de A. argillacea. As larvas de 3o ínstar necessitaram de 4,5 dias (Tabela 1) para chegarem à fase de pré-pupa, com viabilidade de $100 \%$. Este resultado foi semelhante aos obtidos por CARVALHO et al. (1998), de quatro dias e $100 \%$ de viabilidade.

A duração da fase de pré-pupa foi de 1,4 dias (Tabela 1), sendo próxima à observada por NUÑEZ (1988) para $\boldsymbol{C}$. cincta, espécie pertencente a outro gênero, para a qual a duração média da fase pré-pupal foi de um dia quando alimentadas com Sitotroga cerealella. ALBUQUERQUE et al. (1994) encontraram para C. externa duração do período de pré-pupa de 3,3 dias, quando criadas a $23,9^{\circ} \mathrm{C}$. As diferenças nos resultados obtidos nos diversos estudos estão relacionadas à temperatura e à dieta oferecida às larvas de C.externa.

A viabilidade de $86,7 \%$ (Tabela 1), observada para a fase de pré-pupa, foi inferior àquelas observadas por VENZON \& CARVALHO (1993) e CARVALHO et al. (1998), também para C. externa, que obtiveram 100\%, utilizando ovos de $\boldsymbol{A}$. argillacea na dieta.

A duração da fase pupal foi de 9,3 dias (Tabela 1), com 83,1\% de viabilidade, resultado inferior ao obtido por CARVALHO et al. (1998) para C. externa alimentada com ovos de A. argillacea e próximo dos resultados encontrados por MURATA \& DE BORTOLI (2009) para $C$. externa alimentada com o pulgão Brevicoryne brassicae, cujo valor foi de $85 \%$.

A fase larval teve uma duração média de 13,1 dias. Os resultados obtidos por CARVALHO et al. (1998) para C. externa alimentada com ovos de Anagasta kuehniella foi, em média, 11 dias. Para a mesma espécie, DE BORTOLI et al. (2006) observaram desenvolvimento larval de 9,51, 9.37 e 9,02 dias, respectivamente, quando as larvas de $\boldsymbol{C}$. externa receberam como alimentação ovos de Diatraea saccharalis, S. cerealella e A. kuehniella com viabilidade larval de 73,33, 83,33 e 83,33, respectivamente.

A viabilidade larval do $1^{\circ}, 2^{\circ}$ e $3^{\circ}$ instar foi de $96,7 \%, 100 \%$ e $100 \%$, respectivamente (Tabela 1 ). Verificou-se que, das 777 larvas que atingiram o estágio larval, 13,3\% morreram, não confeccionando o casulo. Já a duração do ciclo biológico foi em média de 27,8 dias. Este período foi próximo a 26,93 dias, obtidos por AWADALLAH et al. (1975) para C. carnea, quando alimentada com Thrips tabaci.

O período de oviposição foi de 33,6 dias (Tabela 1), com fecundidade média diária de 11,3 ovos. Os resultados referentes à fecundidade diária foram inferiores aos observados por RIBEIRO \& CARVALHO (1991), que verificaram que C. externa apresentaram uma fecundidade média de 15,86 ovos por dia, quando alimentadas com ovos de A. kuehniella. Algumas fêmeas produziram, no máximo, 444 ovos (Tabela 1) em 50 dias, enquanto que os resultados obtidos por BARBOSA et al. (2000), também com C. externa, foram de, no máximo, 350 ovos, concluindo que fêmeas permanentemente mantidas com os machos produziram um maior número de ovos. MACEDO et al. (2003) observaram que o reacasalamento estimula a oviposição, evitando o esgotamento das reservas espermáticas de fêmeas de $\boldsymbol{C}$. externa.

A longevidade de machos e fêmeas foi próxima. Foi observado, nomínimo, 17 dias para machos e fêmeas e 54 e 53 dias, no máximo, para fêmeas e machos, respectivamente (Tabela 1). Estes resultados de longevidade podem ter ocorrido em virtude da manipulação dos adultos, já que a literatura menciona até 120 dias de vida para insetos adultos (CANARD \& PRINCIPI, 1984).

Para o primeiro ínstar larval, a média do diâmetro da cápsula cefálica foi de $0,48 \mathrm{~mm}$, para o segundo ínstar $0,56 \mathrm{~mm}$ e, para o terceiro ínstar, $0,81 \mathrm{~mm}$ de diâmetro. A razão de crescimento foi de 1,44, podendo assim aplicar a Regra de Dyar. O número de ínstares foi bem evidenciado pelo elevado valor de coeficiente de determinação ( $\mathrm{R}^{2}$ próximos a um) e o valor de razão de crescimento $(\mathrm{K})$ que se encontra dentro dos limites propostos por Dyar (1,1 a 1,9).

O consumo de ovos está relacionado ao estádio larval. De acordo com os valores obtidos, mais de $80 \%$ do alimento fornecido (ovos) foi consumido durante o 3 - ínstar larval, sendo seguido pelas larvas de $2^{\circ}$ e $1^{\circ}$ ínstar, apresentando consumo médio de 429 , 75 e 17 ovos, respectivamente. O consumo médio total da fase larval foi de 520 ovos para que o inseto completasse o ciclo de desenvolvimento, com amplitudes entre 550 e 488 ovos consumidos.

Determinou-se, por meio da tabela de vida e fertilidade, um período médio de geração (T) de 32,69 dias (Tabela 2). Esse valor representa o período 
Tabela 2 - Número médio de dias de oviposição (x), número médio de ovos/fêmea (mx), índice de sobrevivência (lx) e número de descendentes (lx.mx) para a Tabela de Vida de Fertilidade de Chrysoperla externa, alimentadas com ovos de Bonagota cranaodes, com temperatura de $25 \pm 2^{\circ} \mathrm{C}$ e $70 \pm 10 \%$ de umidade relativa e fotofase de 14 horas.

\begin{tabular}{|c|c|c|c|c|c|}
\hline Fase & $\mathrm{X}$ & Mx & $1 \mathrm{x}$ & mx.lx & mx.lx.x \\
\hline Ovo & 0,5 & & 1,00 & & \\
\hline \multicolumn{6}{|c|}{ Larva } \\
\hline \multicolumn{6}{|c|}{ Pré-pupa } \\
\hline \multicolumn{6}{|l|}{ Pupa } \\
\hline & 28,5 & 27,50 & 0,35 & 9,63 & 274,31 \\
\hline & 29,5 & 22,20 & 0,35 & 7,77 & 229,22 \\
\hline & 30,5 & 15,25 & 0,35 & 5,34 & 162,79 \\
\hline & 31,5 & 14,55 & 0,33 & 4,80 & 151,25 \\
\hline & 32,5 & 11,45 & 0,31 & 3,55 & 115,36 \\
\hline & 33,5 & 11,10 & 0,31 & 3,44 & 115,27 \\
\hline & 34,5 & 10,40 & 0,31 & 3,22 & 111,23 \\
\hline & 35,5 & 9,85 & 0,28 & 2,76 & 97,91 \\
\hline & 36,5 & 9,35 & 0,28 & 2,62 & 95,56 \\
\hline A & 37,5 & 8,80 & 0,24 & 2,11 & 79,20 \\
\hline $\mathrm{D}$ & 38,5 & 8,35 & 0,22 & 1,84 & 70,72 \\
\hline $\mathrm{U}$ & 39,5 & 7,20 & 0,21 & 1,51 & 59,72 \\
\hline $\mathrm{L}$ & 40,5 & 3,85 & 0,21 & 0,81 & 32,74 \\
\hline $\mathrm{T}$ & 41,5 & 3,55 & 0,15 & 0,53 & 22,10 \\
\hline \multirow[t]{12}{*}{$\mathrm{O}$} & 42,5 & 3,40 & 0,15 & 0,51 & 21,68 \\
\hline & 43,5 & 2,65 & 0,12 & 0,32 & 13,83 \\
\hline & 44,5 & 2,55 & 0,12 & 0,31 & 13,62 \\
\hline & 45,5 & 1,95 & 0,10 & 0,20 & 8,87 \\
\hline & 46,5 & 0,50 & 0,01 & 0,01 & 0,23 \\
\hline & 47,5 & 0,00 & 0,00 & 0,00 & 0,00 \\
\hline & 48,5 & 0,00 & 0,00 & 0,00 & 0,00 \\
\hline & 49,5 & 0,00 & 0,00 & 0,00 & 0,00 \\
\hline & 50,5 & & & & \\
\hline & Somatório & & & 51,26 & 1675,61 \\
\hline & Ro & 51,26 & & $\mathrm{rm}$ & 0,12044 \\
\hline & $\mathrm{T}$ & 32,69 (dias) & & $\lambda$ & 1,12799 \\
\hline
\end{tabular}

compreendido do nascimento dos pais ao nascimento dos descendentes, ou seja, a duração média de uma geração. Para a população de $\boldsymbol{C}$. externa a taxa máxima de aumento ocorreu no $29^{\circ}$ dia, resultado evidenciado nos valores da taxa de sobrevivência (lx) e da fertilidade específica (mx) (Figura 1).

A taxa líquida de reprodução(Ro) foi de 51,26 vezes (Tabela 2), que indica a estimativa do número médio de fêmeas gerado por fêmea por dia, ao longo do período de oviposição e que chegarão na geração seguinte. $O$ valor da taxa intrínseca de crescimento $\left(\mathrm{r}_{\mathrm{m}}\right)$ foi de 0,12044 , indicando a velocidade de crescimento da população de C. externa. A razão finita de aumento ( $\lambda$ ) foi de 1,12799 , que representa o número de indivíduos adicionados à população por fêmea por um dia.

\section{CONCLUSÃO}

Ovos de Bonagota cranaodes permitem o desenvolvimento das fases imaturas e adulta de Chrysoperla externa e podem ser utilizados para a criação de $\boldsymbol{C}$. externa, entretanto a sua utilização para criação massal se torna inviável pela baixa fecundidade.

\section{AGRADECIMENTOS}

Ao Doutor Gilberto Soares Albuquerque, da Universidade Estadual do Norte Fluminense, pela contribuição na identificação das espécies de crisopídeos. 


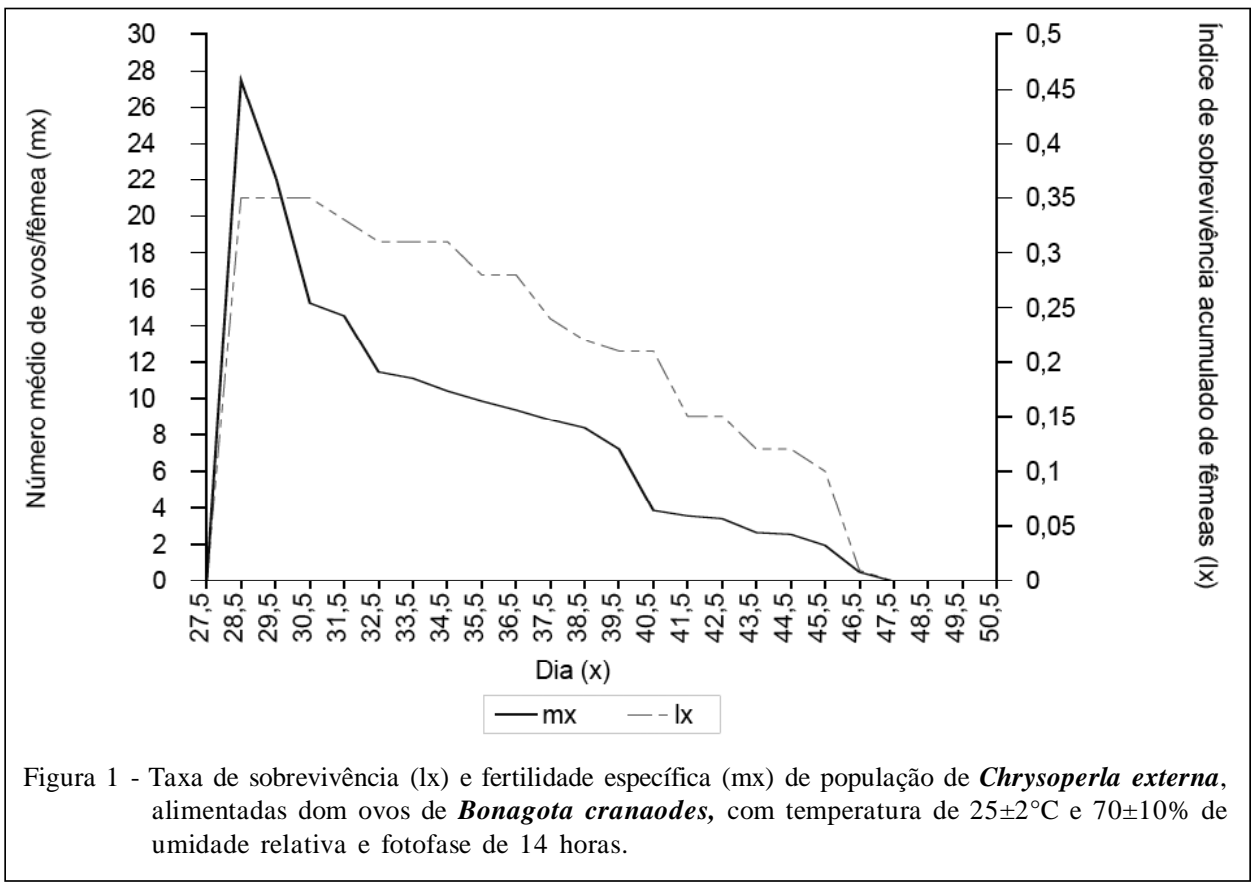

\section{REFERÊNCIAS}

ADAMS, P.A.; PENNY, N.D. Neuroptera of the amazon basin. Part 11 Introductions and chrysopini. Acta Amazonica, v.15, p.413-479, 1987.

ALBUQUERQUE, G.S. et al. Chrysoperla externa (Neuroptera: Chrysopidae). Life history and potential for biological control in Central and South America. Biological Control, v.4, n.1, p.8-13, 1994. Disponível em: <http://www.scielo.br/ scielo.php? script $=$ sci_arttext\&pid=S 0103 $84782008000800002 \& \operatorname{lng}=$ pt\&nrm=iso $>$. Acesso em: 15 jan. 2011. doi: $10.1006 /$ bcon.1994.1002.

AUAD, A.M. et al. Desenvolvimento da fases imaturas, aspectos reprodutivos e potencial de predação de Chrysoperla externa (Hagen) alimentadas com ninfas de Bemicia tabaci (Gennadius) biótipo B em tomateiro. Acta Scientiarum Agronomy, v.27, n.2, p.327-334, 2005. Disponível em: <http://periodicos.uem.br/ ojs/index.php/ActaSciAgron/issue/view/128>. Acesso em: 25 jan. 2011. doi: 10.4025/actasciagron.v27i2.1853.

AWADALLAH, K.T. et al. Development and fecundity of Chrysopa carnea Stephens. Bulletin de la Société Entomologique d'Egypt, v.89, p.323-329, 1975.

BARBOSA, L.R. et al. Capacidade predatória de Chrysoperla externa alimentada com Frankliniella schultzei em plantas de alface de cultivo hidropônico. Revista de Agricultura, Piracicaba, v.75, n.3. p.349-358, 2000.

BIAGIONI, A.; FREITAS, S. Efeito de diferentes dietas sobre o desenvolvimento pós-embrionário de Chrysoperla defreitasi Brooks (Neuroptera: Chrysopidae). Neotropical Entomology, v.30, p.333-336, 2001. Disponível em: <http://www.scielo.br/ scielo.php?script=sci_abstract\&pid=S 1519 $566 \mathrm{X} 2001000200020 \& \operatorname{lng}=\mathrm{pt} \& \mathrm{nrm}=\mathrm{iso} \& \mathrm{t} \operatorname{lng}=\mathrm{pt}>$. Acesso em: 25 jan. 2011. doi: 10.1590/S1519-566X2001000200020.
CANARD, M.; PRINCIPI, M.M. Development of chrysopidae. In: CANARD, M. et al. Biology of Chrysopidae, The Hague: W. Junk, 1984. V.27, 294p.

CARVALHO, C.F. et al. Predation capacity and reproduction potential of Chrysoperla externa (Hagen, 1861) (Neuroptera: Chrysopidae) fed on Alabama argillacea (Hubner) eggs. Acta Zoological Fennica, v.209, p.83-86, 1998. Disponível em: <http://www.sekj.org/ActaZool.html>. Acesso em: 06 dez. 2010.

CARDOSO, J.T. et al. Ocorrência e flutuação populacional de Chrysopidae (Neuroptera) em áreas de plantio de Pinus taeda (L.) (Pinaceae) no Sul do Paraná. Revista Brasileira de Entomologia, v.47, n.3, p.473-475, 2003. Disponível em: $<$ http://www.scielo.br/scielo.php?script=sci_arttext\&pid=S0085$56262003000300019 \& \operatorname{lng}=$ pt\&nr $>$. Acesso em 26 de jan. de 2011. doi: $10.1590 /$ S0085-56262003000300019.

DE BORTOLI, S.A. et al. Desenvolvimento e capacidade predatória de Chrysoperla externa (Hagen, 1861) (Neuroptera: Chrysopidae) em diferentes presas. Revista de Biologia e Ciências da Terra, v.6, n.1, p.145-152, 2006. Disponível em: <http://eduep.uepb.edu.br/rbct/sumarios/sumario_v6_n1.htm>. Acesso em: 01 dez. 2010.

FERREIRA, J.A. et al. Seletividade de inseticidas usados na cultura da macieira a duas populações de Chrysoperla externa (Hagen, 1861) (Neuroptera: Chrysopidae). Ciência Rural, v.36, n.2, p.378-384, 2006. Disponível em: <http:// www.scielo.br/scielo.php? script $=$ sci_arttext $\&$ pid $=$ S0 103 $84782006000200004 \& \operatorname{lng}=$ pt\&nrm=iso $>$. Acesso em $01 \mathrm{dez}$. 2010. doi: 10.1590/S0103-84782006000200004.

FONSECA, A.R. et al. Capacidade predatória e aspectos biológicos das fases imaturas de Chrysoperla externa (Hagen) (Neuroptera: Chrysopidae) alimentada com Schizaphis graminum (Rondani, 1852) (Hemiptera: Aphididae) em diferentes temperaturas. Ciência e Agrotecnologia, v.25, n.2, p.251-263, 2001. 
Disponível em: <http://www.editora.ufla.br/site/_adm/upload/ revista/25-2-2001_03.pdf>. Acesso em 24 nov. 2010.

FREITAS, S.; ADAMS, P. Taxonomia de predadores Chrysopidae (Neuroptera) de ocorrência no Brasil. In: SIMPÓSIO DE CONTROLE BIOLÓGICO, 1994, Gramado-RS. Resumos... Local: EMBRAPA, CPACT, Pelotas, RS, 1994. p.260.

FREITAS, S. Criação de crisopídeos (Bicho lixeiro) em laboratório. Jaboticabal: Fundação de Estudos e Pesquisas FUNEP, 2001. 20p.

FREITAS, S.; PENNY, N.D. The green lacewings (Neuroptera: Chrysopidae) of Brazilian Agro-ecosystems. Proceedings of the California Academy of Sciences, v.52, p.245-395, 2001.

FREITAS, S. O uso de crisopídeos no controle biológico de pragas. In: PARRA, J.R.P. et al. Controle biológico no Brasil: parasitóides e predadores. São Paulo: Manole, 2002. Cap.13, p.209-219.

GRAVENA, S. Manejo integrado de pragas do citrus. Laranja, n.5, p.323-361, 1984.

MAIA, W.J.M.S. et al. Exigências térmicas de Chrysoperla externa (Hagen, 1861) (Neuroptera: Chrysopidae) alimentada com Schizaphis graminum (Rondani, 1852) (Homoptera: Aphididae) em condições de laboratório. Ciência e Agrotecnologia, v.24, p.81-86, 2000. Disponível em: <http:/ /www.editora.ufla.br/site/_adm/upload/revista/24-1 2000_10.pdf>. Acesso em 10 de nov. de 2010.

MACEDO, L.P.M., et al. Influência do fotoperíodo no desenvolvimento e reprodução de Chrysoperla externa (Hagen) (Neuroptera: Chrysopidae). Neotropical Entomology,v.32, p.91-96, 2003. Disponível em: <http://www.scielo.br/ scielo.php? script =sci_arttext \& pid = S 1519 $566 X 2003000100013 \& \operatorname{lng}=$ pt\&nrm=iso $>$. Acesso em 11 de nov. de 2010. doi: 10.1590/S1519-566X2003000100013.

MURATA, A.T.; DE BORTOLI, S.A. Estudo da capacidade de consumo do pulgão da couve por Chrysoperla externa e Ceraeochysa cubana (Neuroptera: Chrysopidae). Revista Brasileira de Agroecologia. v.4, n.2, p.3034-3038, 2009. Disponível em: <http://www.aba-agroecologia.org.br/ojs2/index.php/ rbagroecologia/article/view/8902/6238>. Acesso em: $01 \mathrm{dez} .2010$.
NUÑEZ, Z.E. Ciclo biológico y crianza de Chrysoperla externa e Ceraeochysa cincta (Neuroptera: Chrysopidae). Revista Peruana de Entomologia. v.31, p.76-82, 1988. Disponível em: <http:// www.revperuentomol.com.pe/contenido-y-articulo.html>. Acesso em: 26 nov. 2010.

PARRA, J.R.P. et al. Técnica de criação de Phatheochroa cranaodes Meyrick (Lepidoptera: Tortricidae) em dieta artificial. Revista Brasileira de Biologia, v.55, n.4, p.537$543,1995$.

PARRA, J.R.P.; HADDAD M.L. Determinação do número de instares de insetos. Piracicaba: FEALQ, 1989. 49p.

PENNY, N.D. Lista de Megalopetra, Neuroptera e Raphidioptera do México, América central, Ilhas Caraíbas e America do Sul. Acta Amazônica, v.7, 61p, 1977.

RIBEIRO, M.J. et al. Influência da alimentação larval sobre a biologia de adultos de Chrysoperla externa (Hagen, 1861) (Neuroptera: Chrysopidae). Ciência e Prática, v.15, n.4, p.349-354, 1991

RIBEIRO, M.J.; CARVALHO, C.F. Aspectos biológicos de Chrysoperla externa (Hagen, 1861) (Neuroptera: Chrysopidae) em diferentes condições de acasalamento. Revista Brasileira de Entomologia, v.35, n.2, p.423-427, 1991.

SILVA, G.A. et al. Aspectos biológicos de Chrysoperla externa (Hagen, 1861) (Neuroptera: Chrysopidae) alimentada com lagartas de Alabama argillacea (Hübner, 1818) (Lepidoptera: Noctuidae). Ciência e Agrotecnologia, v.26, n.4, p.682-698, 2002. Disponível em: <http://www.editora.ufla.br/site/_adm/ upload/revista/26-4-2002_03.pdf>. Acesso em: 11 nov. 2010.

SILVEIRA NETO, S. et al. Manual de ecologia dos insetos. São Paulo: Ceres, 1976. 419p.

TULISALO, U. et al. Biological control of aphid with Chrysopa carnea on parsley and green pepper in the greenhouse. Annales Entomologici Fennici, v.43, p.97-100, 1977.

VENZON, M.; CARVALHO, C.F. Desenvolvimento larval, prépupal e pupal de Ceraeochrysa cubana (Hagen) (Neuroptera, Chrysopidae) em diferentes dietas e temperaturas. Anais da Sociedade Entomológica do Brasil. v.22, n.3, p.477-483, 1993. 